\title{
Changes in brain activity of somatoform disorder patients during emotional empathy after multimodal psychodynamic psychotherapy
}

\author{
Moritz de Greck ${ }^{1,2 *}$, Annette F. Bölter ${ }^{3}$, Lisa Lehmann ${ }^{4}$, Cornelia Ulrich ${ }^{5}$, Eva Stockum ${ }^{3}$, Björn Enzi ${ }^{6}$, \\ Thilo Hoffmann ${ }^{7}$, Claus Tempelmann ${ }^{8}$, Manfred Beutel ${ }^{1}$, Jörg Frommer ${ }^{3}$ and Georg Northoff ${ }^{9}$
}

\author{
${ }^{1}$ Department of Psychosomatic Medicine and Psychotherapy, University Medicine Mainz, Mainz, Germany \\ 2 Department of Psychosomatic Medicine and Psychotherapy, University Hospital Leipzig, Leipzig, Germany \\ ${ }^{3}$ Department of Psychosomatic Medicine and Psychotherapy, Otto-von-Guericke-University Hospital, Magdeburg, Germany \\ ${ }^{4}$ Department of Cardiology, Klinikum Bielefeld, Bielefeld, Germany \\ ${ }^{5}$ Department of Psychotherapeutic Medicine, Fachklinikum Uchtspringe, Uchtspringe, Germany \\ ${ }^{6}$ Department of Psychiatry, Ruhr-University Bochum, LWL University Hospital, Bochum, Germany \\ 7 Department of Psychotherapeutic Medicine, Diakoniewerk Halle, Halle, Germany \\ ${ }^{8}$ Department of Neurology, Otto-von-Guericke University Hospital, Magdeburg, Germany \\ ${ }^{9}$ Mind, Brain Imaging and Neuroethics Unit, Institute of Mental Health Research, University of Ottawa, Ottawa, ON, Canada
}

\section{Edited by:}

Nikolai Axmacher, University of Bonn, Germany

\section{Reviewed by:}

Henrik Kessler, University of Bonn Germany

Samantha J. Brooks, Uppsala

University, Sweden

*Correspondence:

Moritz de Greck, Department of

Psychosomatic Medicine and

Psychotherapy, University Medicine,

Untere Zahlbacher Str. 8, 55131

Mainz, Germany

e-mail:moritz.greck@gmx.de
Somatoform disorder patients show a variety of emotional disturbances including impaired emotion recognition and increased empathic distress. In a previous paper, our group showed that several brain regions involved in emotional processing, such as the parahippocampal gyrus and other regions, were less activated in pre-treatment somatoform disorder patients (compared to healthy controls) during an empathy task. Since the parahippocampal gyrus is involved in emotional memory, its decreased activation might reflect the repression of emotional memories (which-according to psychoanalytical concepts-plays an important role in somatoform disorder). Psychodynamic psychotherapy aims at increasing the understanding of emotional conflicts as well as uncovering repressed emotions. We were interested, whether brain activity in the parahippocampal gyrus normalized after (inpatient) multimodal psychodynamic psychotherapy. Using $\mathrm{fMRl}$, subjects were scanned while they shared the emotional states of presented facial stimuli expressing anger, disgust, joy, and a neutral expression; distorted stimuli with unrecognizable content served as control condition. 15 somatoform disorder patients were scanned twice, pre and post multimodal psychodynamic psychotherapy; in addition, 15 age-matched healthy control subjects were investigated. Effects of psychotherapy on hemodynamic responses were analyzed implementing two approaches: (1) an a priori region of interest approach and (2) a voxelwise whole brain analysis. Both analyses revealed increased hemodynamic responses in the left and right parahippocampal gyrus (and other regions) after multimodal psychotherapy in the contrast "empathy with anger" - "control." Our results are in line with psychoanalytical concepts about somatoform disorder. They suggest the parahippocampal gyrus is crucially involved in the neurobiological mechanisms which underly the emotional deficits of somatoform disorder patients.

\section{Keywords: psychodynamic, psychotherapy, fMRI, emotional empathy, somatoform disorder}

\section{INTRODUCTION}

Somatoform disorders contain a group of complex diseases consisting of medically unexplained somatic symptoms (Kirmayer et al., 1994; Stein and Muller, 2008; Pedrosa Gil et al., 2009; Hiller et al., 2010).

Psychologically, they are linked to alexithymia, a construct which describes decreased emotional awareness (Sifneos, 1973; Bach and Bach, 1996; Bankier et al., 2001; Duddu et al., 2003; Grabe et al., 2004; Burba et al., 2006; Bailey and Henry, 2007; Mattila et al., 2008; Pedrosa Gil et al., 2008; Wood et al., 2009). In addition, another emotional process-emotion recognition (i.e., the correct labeling of emotions, Pedrosa Gil et al. 2009)— is impaired (Pedrosa Gil et al., 2009; de Greck et al., 2012), and somatoform disorder patients describe increased "empathic distress" (i.e., they experience themselves as being easily affected and overwhelmed by negative emotional states of others, Davis 1983; de Greck et al. 2012).

From a psychodynamic perspective, emotional alterations of somatoform patients are interpreted as being caused by the unconscious repression of specific emotions to avoid interpersonal conflicts, which would cause strong negative affects (Bowlby, 1973; Waller and Scheidt, 2006). Somatizing patients are 
thus unable to verbally express their emotional states whilst they still experience the somatic component related to their affective reaction (as symptom, Schur, 1955; Krystal, 1997; Beutel et al., 2008). In addition, increased attention to body sensations (in order to distract from interpersonal conflicts) plays an important role (Eriksen and Ursin, 2004; Nakao and Barsky, 2007; Witthöft and Hiller, 2010; de Greck et al., 2011).

Neurophysiologically, as was demonstrated in a previous paper by our group, pre-treatment somatoform disorder patients show diminished modulation of neuronal activity in several brain regions including the bilateral parahippocampal gyrus, the left amygdala, the left postcentral gyrus, the left superior temporal gyrus, and the left posterior insula, during emotional empathy (compared to healthy control subjects, de Greck et al. 2012). In particular, diminished neuronal activation of the parahippocampal gyrus is highly interesting, since other studies emphasized the crucial role of this region in the recall of autobiographical memories (Maguire, 2001; Niki and Luo, 2002; Rekkas and Constable, 2005; Gardini et al., 2006). Especially the retrieval of emotional memories activates the parahippocampal gyrus: For instance, Damasio and colleagues showed that the parahippocampal gyrus is involved in the induction of emotion by intentional retrieval of autobiographic emotional memory (Damasio et al., 2000). In addition, Smith and colleagues (2004) and Sterpenich and colleagues (2006) showed that the parahippocampal gyrus is also involved in the retrieval of emotional background contexts during the active recall of memorized neutral stimuli. Even more interesting, two studies found evidence showing that the parahippocampal gyrus is particularly involved in the processing of conflict related memories: Loughead and colleagues (2010) investigated brain activity during the recall of autobiographic relationship episodes, while also checking for relationship conflict. They found that activity in the parahippocampal gyrus was positively correlated with the degree of conflict related to autobiographical episodes. In complement to this, Schmeing and colleagues (2013) reported a deactivation of the parahippocampal cortex during free associations to conflict-related sentences (when compared to neutral sentences). Subsequent to the free association task, the authors included an unexpected memory recall task. They found that free associations to conflict-related sentences were more often forgotten when compared to free associations made to neutral sentences.

The diminished activation of the parahippocampal gyrus found during emotional empathy in somatoform disorder patients might hence reflect the disturbed retrieval of repressed emotional memories; accordingly, it might be a neurobiological correlate of repression.

Psychodynamic psychotherapy is an established therapy in the treatment of somatoform disorder (and other mental disorders, Leichsenring 2005; Gemeinsamer Bundesausschuss 2009). In the treatment of somatoform disorders, psychodynamic psychotherapy aims to increase the insight and acceptance of unconscious needs and emotional conflicts which underly the client's symptoms (Blagys and Hilsenroth, 2002; Leichsenring, 2005); thus, interpretations are a key instrument (Crits-Christoph et al., 1988). The process of "working through" aims to enable patients to utilize other (namely "healthier") coping strategies (Vaillant,
1977; Wöller and Kruse, 2010), leading to less somatic symptoms and a more satisfying life-style. Further aims of psychodynamic psychotherapy in somatoform disorder include enhancement of the mentalization function, improvement of affect perception and the reduction of medication abuse (Beutel et al., 2008).

Aim of this study was to investigate whether neuronal activity in the parahippocampal gyrus normalized after psychotherapy (mediated by the uncovering of repressed emotional memories). In addition, we were also interested, whether any of the other regions with diminished neuronal activity in the pre-treatment stage (i.e. the left amygdala, the left postcentral gyrus, the left superior temporal gyrus, and the left posterior insula) showed a normalization of neuronal activity in the post-treatment stage.

\section{METHODS}

\section{ETHICAL APPROVAL}

The study was ethically approved by the Institutional Review Board of the Otto-von-Guericke University of Magdeburg/Germany. After a detailed explanation of the study, all subjects gave informed consent. All subjects received financial compensation for their participation in the study. The study was conducted at the Otto-von-Guericke University of Magdeburg/Germany.

\section{PARTICIPANTS}

We investigated 15 patients ( 8 females, 7 males; 14 right handed, 1 left handed; mean age: 42.6 years, $95 \%$-confidence interval: 35.0 50.1 years) suffering from a somatoform disorder as ascertained by the Structured Clinical Interview for DSM-IV (German version: SKID, Wittchen et al. 1997). 11 of the 15 patients fulfilled criteria of an undifferentiated somatoform disorder (DSM-IV: 300.82), 2 of the 15 patients had a somatization disorder (DSMIV: 300.81 ), and 2 of the 15 patients had a pain disorder (DSMIV: 307.80). Leading symptoms of the pre-treatment patients included different forms of pain (e.g., back pain, neck pain, headache), abdominal disturbances (e.g., diarrhea, flatulence, abdominal pain), sexual dysfunctions, and others. All patients were recruited at the start of an inpatient psychotherapy. Patients were recruited from the Department of Psychosomatic Medicine and Psychotherapy of the Otto-von-Guericke-University Hospital in Magdeburg (9/15), from the Department of Psychotherapeutic Medicine of the Fachklinikum Uchtspringe (3/15), and from the Department of Psychosomatic Medicine and Psychotherapy of the AWO Hospital Jerichow (3/15). All patients underwent a second $\mathrm{fMRI}$ session at the end of their psychotherapy. The time difference between both scanning session was 58 days on average (95\%-CI: 51-65 days; range: 38-80 days). During the first fMRI session, 5 patients were on psychotropic medication with duloxetine. During the second fMRI session, one patient continued with duloxetine and one other patient continued with duloxetine and trimipramine. In addition to the patient group, we also investigated 15 gender matched and age matched healthy control subjects ( 8 females, 7 males; 12 right handed, 1 left handed, 2 ambidextrous; mean age: 37.0 years, 95\%CI: $34.4-45.4$ years, $\left.t_{(28)}=0.614 ; p_{\text {[two-tailed] }}=0.545\right)$. This study included 15 patients, who were scanned in their pre-treatment stage and in their post-treatment stage. These 15 patients were taken out of a 
larger population of 20 patients who were scanned in in the pretreatment stage. Due to different reasons, however, 5 of those 20 patients were not scanned in their post-treatment stage. Drop out reasons included premature termination of psychotherapy $(3 / 20)$, refusal to participate a second time $(1 / 20)$, or inaccessibility after discharge $(1 / 20)$.

In addition, this study is part of a larger trial where we compared patients with somatoform disorder patients pre and post multimodal inpatient psychodynamic treatment and healthy control subjects. Data obtained in this trial have already been presented in two previous papers of our group: In one study, we investigated 20 pre-treatment somatoform patients and 20 healthy subjects using the same paradigm (de Greck et al., 2012). In another study, we investigated 20 pre-treatment somatoform patients, 15 post-treatment somatoform patients, and 20 healthy subjects using a different paradigm (de Greck et al., 2011). In the here presented study, we focus on the effects of multimodal inpatient psychodynamic treatment on brain activity of somatoform disorder patients during emotional empathy - these data have not been published before.

\section{PSYCHOTHERAPEUTIC INTERVENTION}

All patients participated in a standardized inpatient multimodal psychodynamic psychotherapy (henceforth "psychotherapy"), which was conducted as recently explained (Grabe et al., 2008; Haase et al., 2008; Huber et al., 2009; de Greck et al., 2011). The therapeutic setting was multimodal and included psychodynamic individual therapy, psychodynamic group therapy, medical therapy, and other therapeutic methods including music therapy, communicative movement therapy, art therapy, social therapy, and relaxation methods (see the Supplementary Material for a more detailed explanation of the therapeutic techniques). Psychotherapy aimed to improve the verbalization of emotional and interpersonal problems, to improve affect perception, and to enhance the understanding of intra-psychic and interpersonal conflicts underlying the patient's symptoms. (Leichsenring, 2005; Beutel et al., 2008; Grabe et al., 2008). Thereby, psychotherapy aimed to enable the patient to utilize a broader spectrum of coping strategies (Vaillant, 1977).

\section{PSYCHOLOGICAL SCALES}

Several psychological measurements were used to investigate differences between patients and healthy subjects as well as differences between patients in the pre-treatment and post-treatment stage. Psychological data of healthy subjects were assessed only once.

Somatization was assessed by the respective sub-scale of the "Symptom Check List 90 - Revised Version" (SCL-90-R, German edition, Derogatis, 1977; Franke, 2002), a self-report questionnaire, which contains several sub-scales. SCL-90 somatization scores were collected from 15 of the 15 pre-treatment somatoform patients, 12 of the 15 post-treatment somatoform patients, and 14 of the 15 healthy control subjects.

Emotional awareness was tested by the German version of the well established self-report questionnaire "Toronto Alexithymia Scale - 20" (TAS-20, Bagby et al., 1994; Bressi et al., 1996). TAS20 scores were collected from the 15 pre-treatment somatoform patients, the 15 post-treatment somatoform patients, and the 15 healthy control subjects.

Mood state and in particular depressive symptoms were assessed with a German edition of the "Beck Depression Inventory" (BDI, Beck et al., 1961). BDI scores were ascertained from the 15 pre-treatment somatoform patients, the 15 post-treatment somatoform patients, and the 15 healthy control subjects.

Emotion recognition abilities were tested using the "Tübinger Affekt Batterie" (TAB, Breitenstein et al. 1998), the German version of the "Florida Affect Battery" (FAB, Bowers et al., 1999). We applied four sub-tests of the TAB: TAB3 and TAB5, which use emotional face stimuli, TAB8a, which uses spoken emotional sentences to test for the ability to identify prosody and semantic content, and TAB8b, which uses spoken sentences as the TAB8a, but applies a number of incongruent auditory stimuli (i.e., sentences with different prosodic and emotional content). The average error rate of all sub-tests was included into the analysis. TAB scores were obtained from 15 pre-treatment somatoform patients, 14 post-treatment somatoform patients, and 12 healthy control subjects.

Statistical analyses of psychological scales included paired samples t-tests to investigate potential effects of psychotherapy, and Spearman-correlations to check for correlations between different scales. Spearman correlations (and not Pearson correlations) were used with regard to the non-linear characteristics of the different scales. We implemented one-tailed tests if we had a directed a priori hypothesis (e.g., improvement of symptom scores in the post-treatment stage), and two-tailed tests otherwise (e.g., differences between two conditions).

\section{PARADIGM}

The paradigm contained a combination of two tasks, a reward anticipation task and an empathy task, which were separated from each other in a block wise manner. Here we report only results obtained from the empathy blocks; please, see our previous paper for results obtained during the reward anticipation paradigm (de Greck et al., 2011).

\section{EXPERIMENTAL DESIGN}

Please also refer to our previous paper for an in depth description (de Greck et al., 2012). Subjects read detailed information about the paradigm and completed a couple of trial runs in order to familiarize with the experiment. In the scanner, stimuli were projected onto a matt screen via an LCD projector, which was visible through a mirror mounted on the head coil. During the experiment three empathy blocks were presented. Each block started with a short finger tapping task. Directly afterwards the actual empathy session began with the presentation of a short instruction, which lasted for $6 \mathrm{~s}$. A total number of 40 empathy trials were then presented in a random order. After every 8 empathy trials, a short pause occurred, lasting for 6,7 , or $8 \mathrm{~s}$ duration; during pauses, the fixation cross was presented. At the end of each block, subjects were asked to rate their present feeling for contentedness as well as their impression of engagement in the empathy task, by moving a bar on a visual analogue scale. Each trial began with the display of an emotional face or a control 
stimulus lasting for $5 \mathrm{~s}$. Subjects were instructed to empathize with the presented emotional face, which was expressed by the phrase "please try to share the emotional state of the person shown." Immediately after the presentation of the emotional face, subjects were asked to rate their ability to empathize with the preceding picture by moving a bar of a visual analogue scale. Prior to the following empathy trial a short inter trial interval (lasting for 2 or $3 \mathrm{~s}$ ) was presented. Facial stimuli expressing the emotion conditions anger, disgust, joy, and neutral emotional state were implemented. Smoothed pictures with unrecognizable contents served as control stimuli.

\section{STIMULI}

The emotional face stimuli were taken from two batteries: the "Japanese and Caucasian Facial Expressions of Emotion"-battery (JACFEE) and the "Japanese and Caucasian Neutral Faces"battery (JACNeuF), both provided by Matsumoto and Ekman (Matsumuto and Ekman, 1988). Eight different facial stimuli of every emotion condition (anger, disgust, joy and neutral) were shown, resulting in 32 different stimuli. Stimuli depicted 16 Caucasian and 16 Japanese actors, half of them female, half of them male. 8 smoothed pictures with unrecognizable contents served as control stimuli. Subjects were instructed to rate the smallest empathy amount (zero) after a control stimulus was presented. During the whole experiment each stimulus was presented once in each block, and for three times during the entire experiment.

\section{FUNCTIONAL MAGNETIC RESONANCE IMAGING (fMRI) fMRI data collection}

fMRI data were collected in a 1.5T MR scanner (General Electric Sigma Horizon) using a standard circular polarized head coil. A stack of 23 slices was aligned parallel to the bicomissural plane. During functional runs 320 whole brain volumes were acquired (gradient echo EPI, $T R=2 \mathrm{~s} ; T E=35 \mathrm{~ms}$; flip angle $\alpha=90^{\circ}$; Field of View $=200 \times 200 \mathrm{~mm}$; slice thickness $=5 \mathrm{~mm}$, interslice gap $=1 \mathrm{~mm}$, spatial resolution $=3.125 \times 3.125 \times 5 \mathrm{~mm}$ ). Additionally, a T1 weighted image of every subject was acquired (3D-FSPGR, 60 saggital slices; $T R=8.8 \mathrm{~ms}$; $T E=1.84 \mathrm{~ms}$; flip angle $\alpha=30^{\circ}$; Field of View $=230 \times 173 \mathrm{~mm}$; slice thickness $=$ $2.8 \mathrm{~mm}$, spatial resolution $=2.8 \times 0.898 \times 0.898 \mathrm{~mm}$.

\section{fMRI data analysis}

Image processing and statistical analyses were carried out using the software package AFNI (http://afni.nimh.nih.gov/afni/, Cox 1996). The first five volumes of each functional run were discarded due to saturation effects. All functional images were slice-time corrected with reference to the acquisition time of the first slice and corrected for motion artifacts by realignment to the first volume. The images were spatially normalized to an AFNI-standard-EPI-template ("TT_EPI") and re-sampled to $3 \times 3 \times 3 \mathrm{~mm}$. Finally, all functional images were smoothed with an isotropic $6 \mathrm{~mm}$ full-width half maximum Gaussian kernel. T1-weighted images were normalized to a standard T1-template provided by AFNI (“TT_avg152T1"). For each subject, a voxelwise whole brain analysis was implemented, and regressors of interest were calculated by the convolution of a gamma response function with the according stimulus time functions (Josephs et al., 1997). All relevant periods (i.e., empathy periods, evaluation periods, pauses, and the free interval at the end of each session) were included in the model. In addition, six movement parameters resulting from the motion correction procedure were included as regressors to account for head motion effects. Likewise, nine regressors for the 3rd degree polynomial model of the baseline of each block were included to control for baseline fluctuations. Contrast images were calculated for each subject by employing linear contrasts to the parameter estimates for the regressors of each event (Friston et al., 1995).

This was followed by a second level group statistic, based on two approaches:

Firstly, we performed a statistical analysis of parameter estimates extracted from regions of interest (ROIs). ROIs were taken from a previous paper of our group in which we used the same paradigm (and partially the same subjects, see above) and found diminished modulation of brain activity of pre-treatment somatoform patients in several brain areas (de Greck et al., 2012). When compared to healthy controls, pre-treatment somatoform patients had shown diminished modulation in their hemodynamic responses in 12 regions (i.e., two regions for the contrast ["anger" + "disgust" + "joy" + "neutral expression"] - "control", seven regions for the contrast "anger" - "control", and three regions for the contrast "joy" - "control”). Spherical ROIs (with a radius of $5 \mathrm{~mm}$ ) were defined based on the coordinates of those 12 regions, and mean contrast values of the according contrasts were extracted. Paired samples $t$-tests were implemented to check for significant differences between pre-treatment and post-treatment somatoform patients. With regard to the high number (12) of statistical tests, we applied a Bonferroni-correction to account for the multiple testing problem. Only those statistical results with a $p$-value of less than $0.05 / 12=0.004$ were treated as significant results.

Secondly, we implemented a voxelwise second level randomeffects analysis using paired samples $t$-tests (comparing the 15 somatoform patients in their pre-treatment stage and posttreatment stage) to identify brain regions which showed altered hemodynamic responses after psychotherapy. Again, we were only interested in contrasts, which had revealed a significant difference between pre-treatment somatoform patients and healthy control subjects in our previous study (de Greck et al., 2012) (namely ["anger" + "disgust" + "joy" + "neutral expression"] - "control," "anger" - "control," “joy" - "control”), whereas we did not investigate changes of hemodynamic responses after psychotherapy for the two other contrasts (namely "disgust" "control" and "neutral expression" - "control"). To control for the multiple testing problem (Nichols and Hayasaka, 2003), we calculated family-wise error probabilities based on Monte-Carlosimulations; in addition, small clusters with a size of $\leq 10$ voxels were not respected. The anatomical localization and labeling of significant activations were assessed with reference to the standard stereotactic atlas of Talairach and Tournoux (1988) and by superimposition of the group contrast images on a mean brain generated by an average of normalized T1-weighted image of all patients. 
Since 5 of the 15 patients were on psychotropic medication during either one or both scanning session, we implemented additional fMRI analyses including only data of those 10 participants who were without medication.

To check whether psychotherapy-induced reductions of somatic symptoms were associated with changes of hemodynamic responses in any of our regions of interest (i.e., the regions mentioned above plus significant regions of the voxelwise second level analysis), we implemented Spearman correlations of psychotherapy induced changes in SCL-90-somatization scores and psychotherapy induced changes of contrast values in the ROIs. We decided to implement Spearman correlations (and not Pearson correlations) with regard to the non-linear characteristics of the SCL-90-somatization scale.

Since we presented differences between pre-treatment somatoform patients and healthy subjects in a previous paper (de Greck et al., 2012), we here solely focused on the differences between pre-treatment and post-treatment somatoform patients. Data of healthy subjects are presented nevertheless for illustration purposes.

\section{RESULTS}

\section{BEHAVIORAL RESULTS}

\section{Psychological Scales}

As shown in Figure 1, psychotherapy had a significant effect on all applied outcome scales. Somatization, as assessed with the somatization sub-scale of the SCL-90-R, was significantly reduced after psychotherapy $\left[t_{(11)}=3.564 ; p_{\text {[one-tailed }]}=\right.$ $\left.0.002^{* *}\right]$. Emotional awareness, as assessed with the TAS-20, was significantly enhanced after psychotherapy $\left[t_{(14)}=2.456\right.$; $\left.p_{\text {[one-tailed }]}=0.014^{*}\right]$. In addition, Mood state was controlled using the BDI. After psychotherapy, we found a significant reduction of depressive symptoms $\left[t_{(14)}=5.660 ; p_{\text {[one-tailed }]}=\right.$ $\left.0.001^{* * *}\right]$. Finally, emotion recognition abilities, which were assessed using the TAB, improved; error rates were significantly lower after psychotherapy $\left[t_{(13)}=2.747 ; p_{\text {[one-tailed }]}=0.008^{*}\right]$.

When checking for correlations between different scales, we found that psychotherapy-induced reductions of SCL90-somatization scores correlated with reductions of BDIscores $\left(\rho_{[\text {Spearman }]}=0.558 ; p_{\text {[one-tailed }]}=0.025^{*}\right)$. In addition, psychotherapy induced reductions of TAS-20 scores correlated with reductions of BDI-scores $\left(\rho_{[\text {Spearman }]}=0.723 ; p_{\text {[one-tailed }]}=\right.$ $\left.0.001^{* *}\right)$. However, we did not find correlations of psychotherapy induced changes of SCL-90-somatization scores with changes in the TAS- $20\left(\rho_{[\text {Spearman }]}=0.323 ; p_{\text {[one-tailed }]}=\right.$ 0.306), of SCL-90-somatization changes with changes of error rates in the $\mathrm{TAB}\left(\rho_{[\text {Spearman }]}=-0.145 ; p_{\text {[one-tailed }]}=0.673\right)$, of TAS-20 changes with changes of error rates in the TAB $\left(\rho_{[\text {Spearman }]}=-0.123 ; p_{\text {[one-tailed }]}=0.674\right)$, or $\left(\rho_{[\text {Spearman }]}=\right.$ $\left.0.139 ; p_{\text {[one-tailed] }}=0.637\right)$.

\section{Intra-scanner empathy ratings}

The $2 \times 4$ factorial ANOVA with "psychotherapy" ("pretreatment" vs. "post-treatment") as first within-subjects factor and "emotion" ("anger," "disgust," “joy," and "neutral expression") as second within-subjects factor revealed a significant effect of "emotion" $\left[F_{(3,112)}=13.367 ; p<0.001^{* * *}\right]$, whilst we did not find significant effects for the factor "psychotherapy" $\left[F_{(1,112)}=\right.$ $1.142 ; p=0.288$ ] or the interaction of "psychotherapy" $\times$ "emotion" $\left[F_{(3,112)}=0.586 ; p=0.626\right]$. Post-hoc $t$-tests revealed, that empathy ratings for "anger" trials were significantly higher compared to "disgust" trials $\left[t_{(14)}=4.715 ; p_{\text {[two-tailed] }}<0.001^{* * *}\right]$ and significantly lower compared to "joy" trials $\left[t_{(14)}=5.924\right.$; $p_{\text {[two-tailed] }}<0.001^{*}$ ]. In addition, empathy ratings for "disgust" trials were significantly lower compared to "joy" trials $\left[t_{(14)}=\right.$ $7.880 ; p_{\text {[two-tailed] }}<0.001^{* * *}$ ], and empathy ratings for "neutral expression" trials were significantly lower compared to "joy" trials $\left[t_{(14)}=6.479 ; p_{\text {[two-tailed] }}<0.001^{* * *}\right]$. There were, however, no significant differences between empathy ratings for "anger" and "neutral expression" $\left[t_{(14)}=0.234 ; p_{\text {[two-tailed] }}=0.818\right]$, and between empathy ratings for "disgust" and "neutral expression" $\left[t_{(14)}=1.621 ; p_{[\text {two-tailed }]}=0.127\right]$.

\section{fMRI RESULTS}

\section{ROI based comparison of hemodynamic responses}

In a previous paper, we found 12 brain areas (regions of interest, ROIs) with diminished modulation of hemodynamic responses in pre-treatment somatoform patients compared to healthy control subjects (de Greck et al., 2012). As shown in Table 2, we found significant improvement of hemodynamic responses

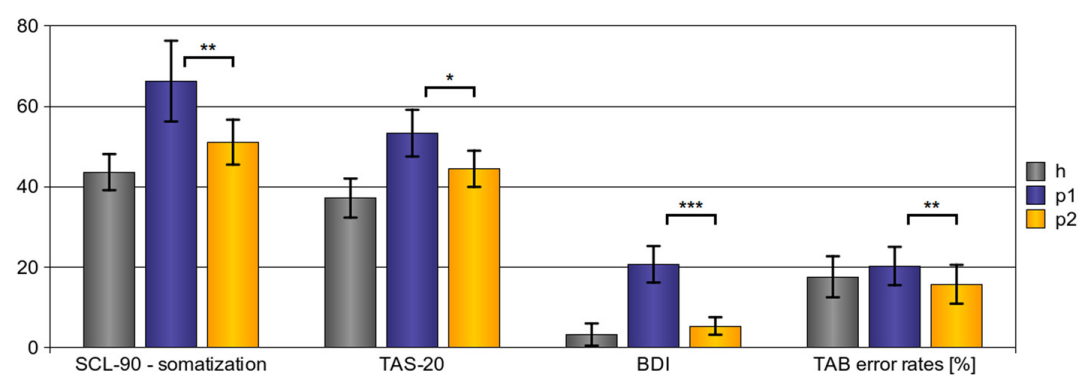

FIGURE 1 | Behavioral results-effects of psychotherapy. After psychotherapy we observed a significant reduction of somatization severity (SCL-90 - somatization scores), to enhanced emotional awareness (TAS-20 scores), and to a reduction of depressive symptoms (BDI scores). In addition, emotion recognition abilities improved after psychotherapy as shown by a significant reduction of error rates in the TAB. (Explanations: $h, p 1$, and $p 2$ refer to the scores of healthy subjects (h), pre-treatment somatoform patients ( $p 1)$, and post-treatment somatoform patients ( $p 2)$; error bars indicate the 95\%-confidence-interval; ${ }^{*} p<0.05,{ }^{* *} p<0.01,{ }^{* * *} p<0.001$, with regard to one-tailed $t$-tests.) 
after psychotherapy in 6 of the 12 regions-all for the contrast "anger"_-control."

\section{Voxel-wise whole brain analysis}

In addition to the above described ROI based approach, we also implemented a voxel-wise whole brain statistical analysis to identify brain regions with altered hemodynamic responses. As presented in Table 3 and Figure 2, four regions showed a significant effect of psychotherapy on hemodynamic modulation: The bilateral parahippocampal gyrus and the left inferior temporal gyrus showed increased modulation of hemodynamic responses after psychotherapy, the left putamen

Table 1 | Intra-scanner empathy ratings.

\begin{tabular}{llll}
\hline Condition & $\boldsymbol{h}$ & $\boldsymbol{p 1}$ & $\boldsymbol{p 2}$ \\
\hline Anger & $62.6 \pm 10.8$ & $62.8 \pm 11.8$ & $63.9 \pm 10.5$ \\
Disgust & $61.2 \pm 12.1$ & $56.9 \pm 12.2$ & $56.8 \pm 10.2$ \\
Joy & $82.8 \pm 8.5$ & $86.7 \pm 5.3$ & $81.3 \pm 7.6$ \\
Neutral expression & $62.0 \pm 9.6$ & $69.2 \pm 8.4$ & $59.8 \pm 9.9$ \\
\hline
\end{tabular}

(Abbreviations: $h, p 1$, and $p 2$ refer to the mean empathy rating of the according condition, where $h$ indicates data of healthy subjects, $p 1$ indicates data of pre-treatment somatoform patients, and p2 indicates data of post-treatment somatoform patients; \pm indicates the $95 \%$-confidence-interval). had diminished modulation of hemodynamic responses after psychotherapy.

\section{Correlation of psychotherapy induced effects}

We were interested, whether psychotherapy induced alleviations of somatic symptoms (as ascertained with the SCL-90somatization sub-scale) correlated with psychotherapy induced changes of hemodynamic responses in any of our ROIs. With regard to those ROIs which had previously shown diminished modulation of hemodynamic responses in pre-treatment somatoform patients (i.e., ROIs listed in Table 1), we did not find any significant correlations under reasonable statistical thresholds (Spearman correlations, $p_{\text {[two-tailed] }}<0.05$ ). With regard to the ROIs found in the voxel-wise analysis (i.e., ROIs listed in Table 2), we found only one significant correlation: the reduction in the SCL-90 somatization scores induced by psychotherapy correlated with the reduction of hemodynamic responses in the left putamen $\left(\rho=0.811 ; p_{\text {[one-tailed] }}=\right.$ $\left.0.001^{* *}\right)$.

\section{Control for potential effects of the psychotropic medication}

Since 5 of the 15 patients were on psychotropic medication during either on one or both scanning sessions, we implemented two additional fMRI analyses (i.e., an additional ROI-based approach and an additional voxelwise approach) including only data of the 10 patients without medication. These results support the

Table 2 | Effect of psychotherapy on hemodynamic responses-ROI based approach.

\begin{tabular}{|c|c|c|c|c|c|c|c|c|}
\hline Region & & \multicolumn{3}{|c|}{ Coordinates } & \multicolumn{3}{|c|}{ fMRI contrast values } & $\begin{array}{c}\text { Psychotherapy effect } \\
\text { p2 }>\text { p1 }\end{array}$ \\
\hline Right & parahippocampal gyrus & 30 & 54 & -3 & $-0.51 \pm 1.77$ & $-5.53 \pm 2.25$ & $-4.26 \pm 2.60$ & $t_{(14)}=0.670 ; p_{\text {[one-tailed] }}=0.257$ \\
\hline Left & amygdala & -24 & -3 & -24 & $0.50 \pm 0.87$ & $-0.59 \pm 1.54$ & $-0.27 \pm 1.47$ & $t_{(14)}=0.355 ; p_{\text {[one-tailed] }}=0.364$ \\
\hline Left & Superior temporal gyrus & -33 & -15 & -27 & $1.05 \pm 0.60$ & $-0.91 \pm 1.03$ & $0.23 \pm 0.94$ & $t_{(14)}=1.899 ; p_{\text {[one-tailed }]}=0.039^{*}$ \\
\hline Left & Parahippocampal gyrus & -33 & 18 & -24 & $0.78 \pm 0.60$ & $-0.39 \pm 0.52$ & $0.19 \pm 0.81$ & $t_{(14)}=1.914 ; p_{\text {[one-tailed }]}=0.038^{*}$ \\
\hline Right & Parahippocampal gyrus & 18 & 21 & -15 & $1.20 \pm 0.91$ & $-0.92 \pm 0.98$ & $0.77 \pm 0.63$ & $t_{(14)}=3.829 ; p_{\text {[one-tailed] }}<0.001 * * * \dagger$ \\
\hline Left & Posterior insula & -36 & 33 & 15 & $0.66 \pm 0.50$ & $-0.56 \pm 0.62$ & $0.12 \pm 0.64$ & $t_{(14)}=2.151 ; p_{\text {[one-tailed] }}=0.025^{*}$ \\
\hline Left & Amygdala & -21 & -3 & -21 & $1.24 \pm 0.83$ & $-0.57 \pm 1.06$ & $0.37 \pm 0.77$ & $t_{(14)}=1.621 ; p_{\text {[one-tailed] }}=0.064^{(*)}$ \\
\hline Right & Cerebellum & 33 & 84 & -27 & $3.70 \pm 1.40$ & $0.31 \pm 1.25$ & $0.65 \pm 1.43$ & $t_{(14)}=0.628 ; p_{\text {[one-tailed] }}=0.270$ \\
\hline Right & Cerebellum & 21 & 87 & -30 & $3.35 \pm 1.44$ & $0.47 \pm 0.95$ & $0.55 \pm 1.01$ & $t_{(14)}=0.159 ; p_{\text {[one-tailed] }}=0.438$ \\
\hline
\end{tabular}

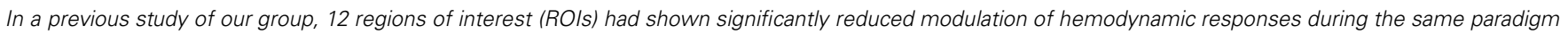

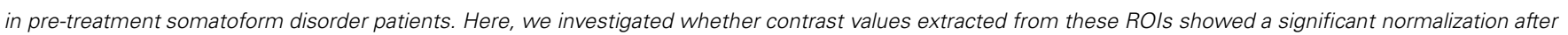

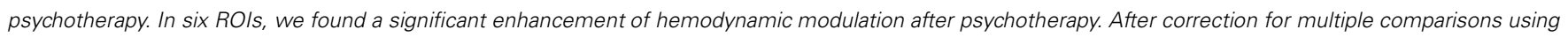

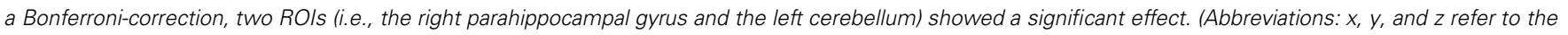

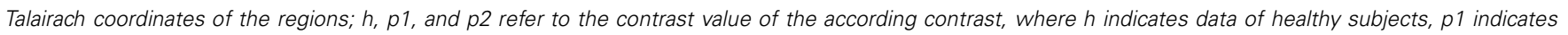

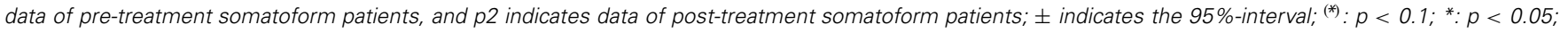
$*^{*}: p<0.01 ;{ }^{* * *}: p<0.001{ }^{+}: p<0.004$, this indicates a significant effect after controlling for multiple comparisons using a Bonferroni-correction.) 
A Left parahippocampal gyrus $[-25,41,-16]$
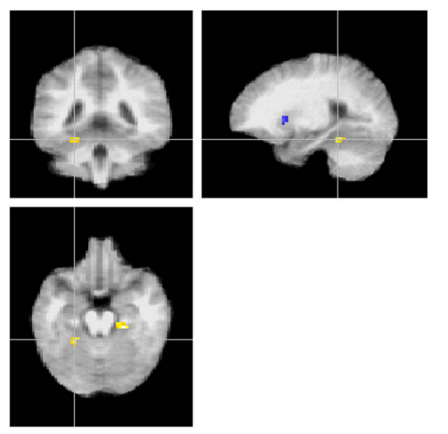

B Right parahippocampal gyrus [22, 26, -16]
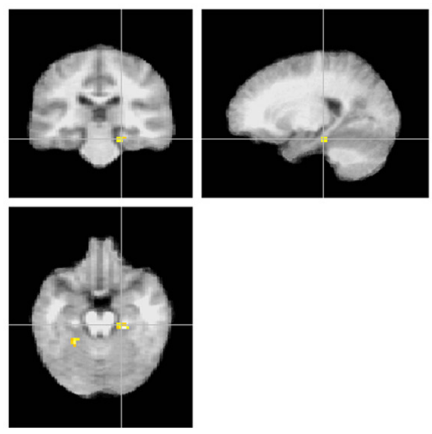

C Left putamen $[-26,-11,5]$
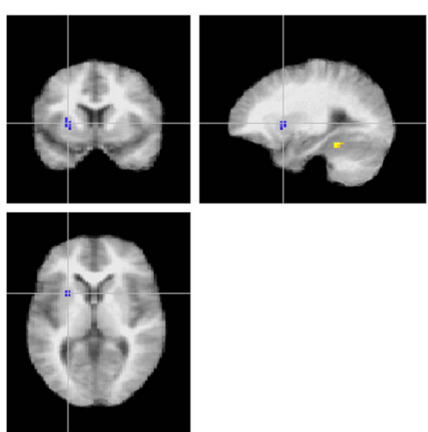

D Left inferior temporal gyrus [-53, -1, -32]
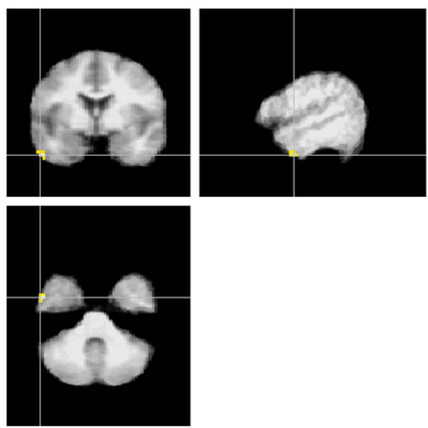

FIGURE 2 | Brain activity modulated by multimodal psychotherapy. Using a voxel-wise whole brain analysis, we found three regions for the contrast "anger"-"control" and one for the contrast "joy"-"control", which had a significant increase (bilateral parahippocampal gyrus, left inferior frontal cortex) or decrease (left putamen) of hemodynamic modulation after psychotherapy. The $p$-threshold was set to $p_{\text {[uncorrected] }} \leq 0.001$; only clusters
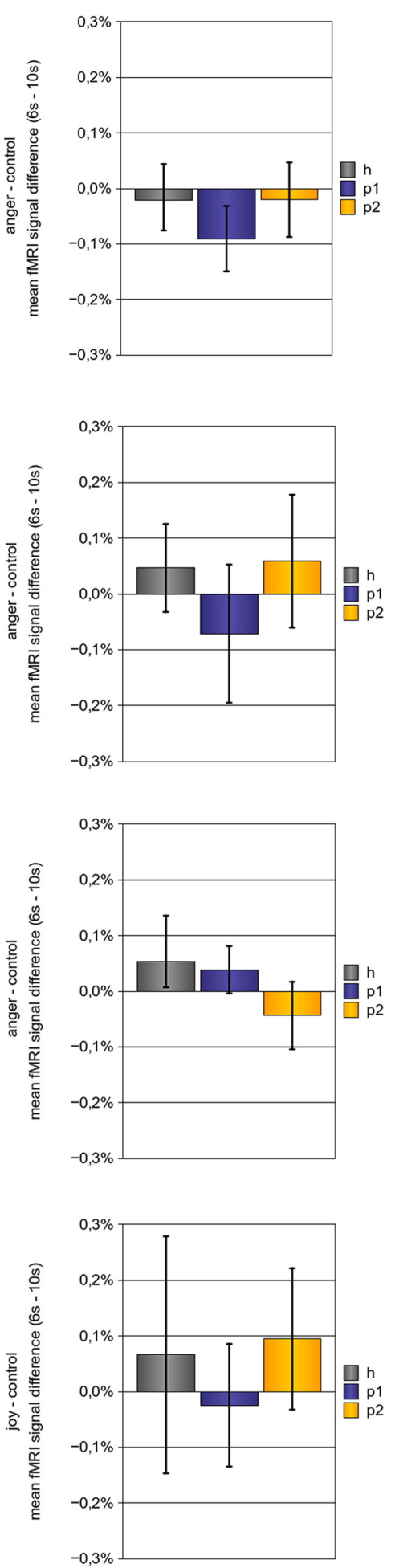

with a cluster size of more than 10 voxels were taken into account. (Abbreviations: $h, p 1$, and $p 2$ refer to the mean $f M R I$ signal difference of the according contrast, where $h$ indicates data of healthy subjects, $p 1$ indicates data of pre-treatment somatoform patients, and $p 2$ indicates data of post-treatment somatoform patients; error bars indicate the 95\%-confidence-interval). 
Table 3 | Effect of psychotherapy on hemodynamic responses-voxel-wise whole brain analysis.

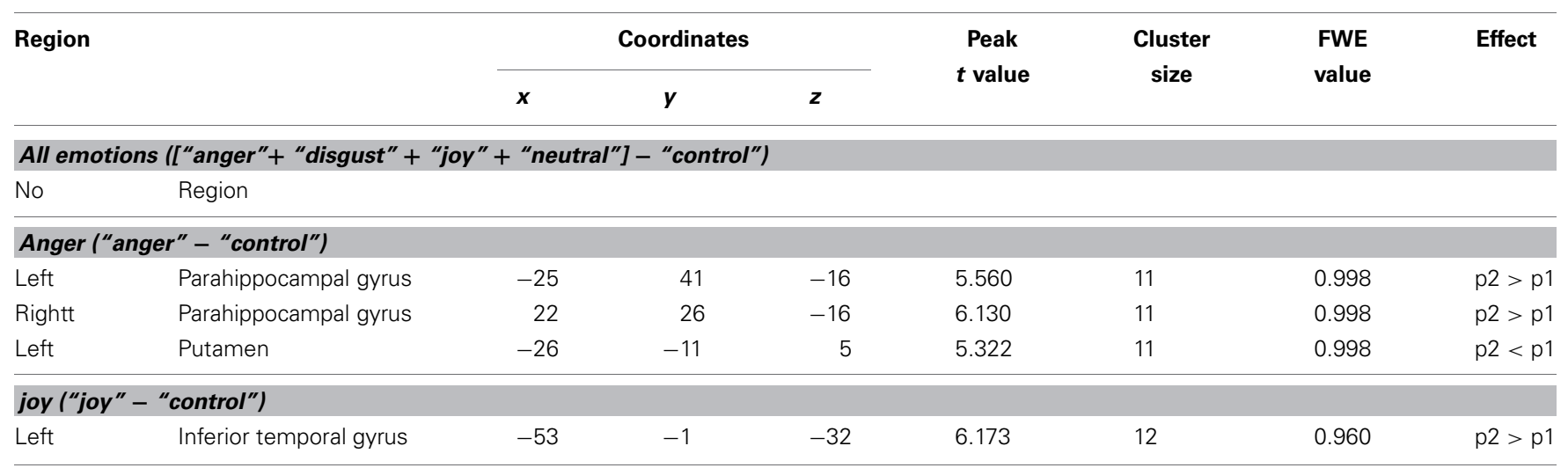

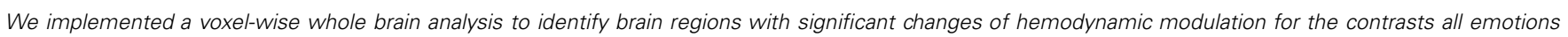

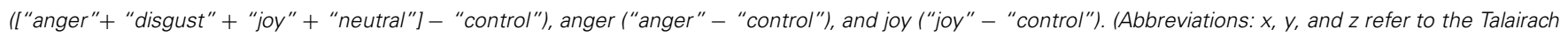

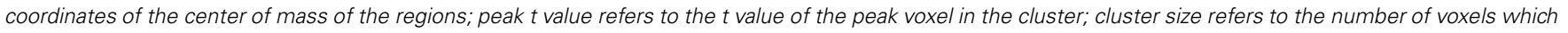

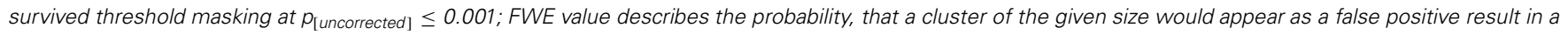
contrast of the given smoothness; effect refers to an increase ( $2>$ p1) or a decrease ( $22<p 1)$ of hemodynamic responses after psychotherapy.)

view that modulation of hemodynamic responses in the left superior temporal gyrus, right parahippocampal gyrus, left posterior insula, and left cerebellum (ROI-based approach), as well as in the in the bilateral parahippocampal gyrus, left putamen, and left inferior frontal gyrus (voxelwise approach) are not caused by the effects of psychotropic medication. Please see the Supplementary Material for a detailed presentation of the according results.

\section{DISCUSSION}

\section{SUMMARY OF FINDINGS}

Somatoform disorder patients in a pre-treatment stage show diminished modulation of hemodynamic responses during emotional empathy in several brain areas, including the bilateral parahippocampal gyrus, left amygdala, left postcentral gyrus and others; this was reported in a previous paper by our group (de Greck et al., 2012). Here, we investigated whether brain activity normalized after multimodal psychodynamic psychotherapy. Psychotherapy was successful as demonstrated by a significant reduction of somatization symptoms (based on the SCL-90 somatization sub-scale), alexithymia symptoms (TAS-20), and depressive symptoms (BDI). In addition, psychotherapy led to significant reduction of error rates in an emotion recognition test (TAB).

The analysis of psychotherapy induced changes of brain activity was implemented using two approaches: a region of interest (ROI) based approach and a voxel-wise whole brain analysis. Both analyses came to the matching results that brain activity in the bilateral parahippocampal gyrus during empathy with anger normalized after psychotherapy. With regard to the ROI based analysis, we found that brain activity in almost all of those regions which had shown diminished modulation of hemodynamic responses in the pre-treatment stage for the contrast "empathy with anger" - "control" normalized after psychotherapy. Regions with a normalization after psychotherapy included the left postcentral gyrus, left superior temporal gyrus, left posterior insula, left amygdala (statistical trend), left cerebellum, and the above mentioned bilateral parahippocampal gyrus.

However, none of those regions which had shown diminished modulation of hemodynamic responses of pre-treatment somatoform patients during other contrasts (namely "empathy with all emotions" - "control," and "empathy with joy" - "control"), showed a normalization of hemodynamic responses after psychotherapy.

With regard to the voxel-wise whole brain analysis, we found three regions, which had a significant change in neuronal activity after psychotherapy for the contrast "empathy with anger" - "control" (the bilateral parahippocampal gyrus, and the left putamen), and one region for the contrast "empathy with joy" - "control" (the left inferior temporal gyrus). Interestingly, the left putamen showed less modulation of hemodynamic responses after psychotherapy, whereas modulation was increased in all other regions. In addition, the left putamen was the only region in which reduction of hemodynamic responses was correlated with the reduction of somatic symptoms after psychotherapy (over all patients).

\section{PSYCHODYNAMIC MECHANISMS IN SOMATOFORM DISORDER}

From a psychodynamic perspective, the development of somatoform symptoms can be understood as malfunctioning of secondary process mechanisms concerning the handling of emotional conflicts which leads to "resomatization" (i.e., the appearance of somatoform symptoms as "concomitants" or "equivalents" of affective tensions, Schur, 1955). In other words: unreleased affective tensions caused by unconscious emotional conflicts induce the corresponding somatic responses (which appear as somatic symptoms), whereas the corresponding affective component is repressed and can not be experienced (Lipowski, 1988; Hoffmann et al., 2004). In this regard, somatization (i.e., the development of medically unexplained somatic symptoms, Lipowski, 1988) is either seen as a defense mechanism itself (Bond et al., 1983; Lipowski, 1988), or as a correlate of immature defense styles (Nickel and Egle, 2006). In addition to 
emotional conflicts, the experience of overwhelmingly strong negative emotional reactions, which may occur for instance as a response to childhood adversities, can lead to a breakdown of secondary process thinking and a malfunction of mature defense mechanisms (Nickel and Egle, 2001). As a consequence, these patients are prone to develop reduced emotional awareness as correlate of the repressed emotional component, and somatic symptoms as equivalents of affective tensions (Krystal, 1997). Indeed, there is a strong link between childhood adversities and adult somatization (Spitzer et al., 2008).

\section{HOW DOES PSYCHOTHERAPY HELP PATIENTS WITH SOMATOFORM DISORDERS?}

Psychodynamic psychotherapy, as it was applied in our study, aims to increase the insight and acceptance of unconscious emotions, needs and conflicts (Blagys and Hilsenroth, 2002; Leichsenring, 2005), and to enable patients to gain "mastery over his or her repressed wishes, desires, fears, or anxieties" (Blagys and Hilsenroth, 2002). Regarding this, the development of more mature defense mechanisms and coping strategies is a core aim (Vaillant, 1977). Further treatment goals include the establishment of a psychosomatic disease model, the enhancement of affect differentiation, a better understanding of underlying stresses, and the reduction of medication abuse (Beutel et al., 2008). In the case of somatoform disorder, successful psychotherapy leads to enhanced secondary process thinking, more mature defense mechanisms, improved "desomatization", and decreased somatic symptoms.

The reduction of somatic symptoms after psychotherapy in our study reflects a decreased use of somatization (i.e., improved "desomatization"). Improved emotion recognition after psychotherapy is probably caused by the uncovering of repressed emotional conflicts and emotional needs, which hampered emotion recognition in the pre-treatment stage.

\section{NEUROPHYSIOLOGICAL MECHANISMS IN SOMATOFORM DISORDER}

The bilateral parahippocampal gyrus seems to play a key role in the affective dysfunctions of somatoform disorder (in particular in the processing of angry facial stimuli), since we found a significantly diminished modulation of hemodynamic responses in this area in the pre-treatment stage (de Greck et al., 2012) and a significant improvement after psychodynamic psychotherapy. Interestingly, the parahippocampal gyrus is involved in the recall of autobiographical memories (Maguire, 2001; Niki and Luo, 2002; Rekkas and Constable, 2005; Gardini et al., 2006), in particular the retrieval of emotional memories (Damasio et al., 2000), or emotional background informations (Smith et al., 2004; Sterpenich et al., 2006). In addition, the recall of conflictual memory content activates the parahippocampal gyrus (Loughead et al., 2010), and it is less activated during free associations to conflict-related themes (Schmeing et al., 2013).

\section{AFFECTIVE PROCESSING IN SOMATOFORM DISORDER AND THE EFFECT OF PSYCHOTHERAPY}

High co-morbidity of somatoform disorder and alexithymia (i.e. diminished awareness of own and other's emotional processes) has been reported by various investigators (Bach and Bach, 1996;
Bankier et al., 2001; Duddu et al., 2003; Grabe et al., 2004; Burba et al., 2006; Bailey and Henry, 2007; Mattila et al., 2008; Wood et al., 2009). From a psychodynamic point of view, the link between alexithymia and somatization is explained by a breakdown of secondary process coping with emotional conflicts, followed by the repression of the corresponding affective component and the experience of the somatic component as unexplained body symptoms (Schur, 1955; Lipowski, 1988; Hoffmann et al., 2004). Our data suggests that the parahippocampal gyrus, a region known for its involvement in the processing of autobiographic emotional memories, might be a neural key correlate of this process. In our study, we investigated brain activity during the intentional empathic processing of facial emotions. The process of intentional emotional empathy rests upon emotional sub processes which include emotional and cognitive empathy, emotion recognition, affective and cognitive mentalizing and the processing of autobiographical memory (Shamay-Tsoory, 2011). In order to empathize with somebody else, it is essential to generate a congruent emotional image of the target's emotional state within oneself (Preston and de Waal, 2002). Besides emotional contagion (Preston and de Waal, 2002; Shamay-Tsoory, 2011), this process relies on the recall of autobiographic memory traces (Damasio et al., 2000): If I want to understand from your face, what you feel, and intentionally want to share your emotional state, I may try to recall own memory traces which previously led to a similar emotional response in myself. This process involves the retrieval of emotional autobiographic memory and induces activity in the parahippocampal gyrus. In the case of somatoform disorder, however, it is assumed that overwhelmingly strong emotional conflicts led to a breakdown of emotional processing and a suppression of the corresponding memory traces, resulting in a diminished modulation of the parahippocampal gyrus during emotional empathy. Psychodynamic psychotherapy, however, can restore the retrieval of repressed autobiographic memories, which leads to increased modulation of the parahippocampal gyrus.

\section{PSYCHOANALYTICAL NEUROSCIENCE}

We believe our study is a fine example, which demonstrates how neuroscience can benefit from psychoanalysis. Our main finding (i.e., pre-treatment somatoform disorder patients show diminished modulation of neuronal activity in the parahippocampal gyrus during the empathic processing of angry faces; this pattern normalizes after psychotherapy) fits well to psychoanalytical concepts of somatoform disorder. In fact, it is hard to explain our findings without referencing to psychodynamic concepts, which relate somatoform disorder to repressed emotional memories.

When it comes to the investigation of the neuronal underpinnings of complex emotional processing (and in particular its dysfunctions in psychosomatic diseases such as somatoform disorder) psychoanalytical concepts may provide a profound base to interpret neuroscientific findings.

\section{LIMITATIONS}

Our psychotherapeutic intervention was "multimodal", i.e., it included a variety of different therapeutic techniques. Hence, we can not conclude for sure that increased emotional insight in 
formerly repressed conflicts caused the reduction of symptoms and normalization of brain activity (and not for instance other therapeutic variables such as decreased depression).

Even more important, with the existing design, we can not for sure conclude that changes observed in the pre-post comparison are indeed caused by psychotherapy since they might be related to other factors, such as for instance retest effects, spontaneous remission, or in particular, regression to the mean effects (Barnett et al., 2005). To control for these potential confounds it would have been essential to scan a second control group consisting of somatoform patients, who would have been also scanned for two times without participation in psychotherapy.

However, the fact that we found reduced neuronal activity in the bilateral parahippocampal gyrus in the pre-treatment stage, and normalized activity in the bilateral parahippocampal gyrus in the post-treatment stage, supports the view that these activations are not related to regression to the mean effects (because it would be rather improbable that regression to the mean effects lead to bilateral effects in the same brain region).

\section{CONCLUSION}

Our results are in accordance with the conclusion that the repression of emotional memories, which occurs in

\section{REFERENCES}

Bach, M., and Bach, D. (1996). Alexithymia in somatoform disorder and somatic disease: a comparative study. Psychother. Psychosom. 65, 150-152. doi: 10.1159/000289067

Bagby, R. M., Parker, J. D., and Taylor, G. J. (1994). The twenty-item toronto alexithymia scale-i. item selection and cross-validation of the factor structure. J. Psychosom. Res. 38, 23-32. doi: 10.1016/00223999(94)90005-1

Bailey, P. E., and Henry, J. D. (2007). Alexithymia, somatization and negative affect in a community sample. Psychiatry Res. 150, 13-20. doi: 10.1016/j.psychres.2006.05.024

Bankier, B., Aigner, M., and Bach, M. (2001). Alexithymia in dsm-iv disorder: comparative evaluation of somatoform disorder, panic disorder, obsessive-compulsive disorder, and depression. Psychosomatics 42, 235-240. doi: 10.1176/appi.psy.42. 3.235

Barnett, A. G., van der Pols, J. C., and Dobson, A. J. (2005). Regression to the mean: what it is and how to deal with it. Int. J. Epidemiol. 34, 215-220. doi: 10.1093/ije/dyh299

Beck, A. T., Ward, C. H., Mendelson, M., Mock, J., and Erbaugh, J. (1961). An inventory for measuring depression. Arch. Gen. Psychiat. 4, 561-571. doi: 10.1001/archpsyc.1961.01710120031004

Beutel, M. E., Michal, M., and Subic-Wrana, C. (2008).
Psychoanalytically-oriented inpatient psychotherapy of somatoform disorders. J. Am. Acad. Psychoanal. Dyn. Psychiatry 36, 125-142. doi: 10.1521/jaap.2008.36.1.125

Blagys, M. D., and Hilsenroth, M. J. (2002). Distinctive activities of cognitive-behavioral therapy. a review of the comparative psychotherapy process literature. Clin. Psychol. Rev. 22, 671-706. doi: 10.1016/S0272-7358 (01)00117-9

Bond, M., Gardner, S. T., Christian, J., and Sigal, J. J. (1983). Empirical study of self-rated defense styles. Arch. Gen. Psychiat. 40, 333-338.

Bowers, D., Blonder, L. X., and Heilman, K. M. (1999). Florida Affect Battery: Center for Neuropsychological Studies. Gainesville, FL: Cognitive Neuroscience Laboratory, University of Florida.

Bowlby, J. (1973). Attachment and Loss. Vol. II. Separation. Anxiety and Anger. London: The Travistock Institute of Human Relations.

Breitenstein, C., Daum, I., and Ackermann, H. (1998). Emotional processing following cortical and subcortical brain damage: contribution of the fronto-striatal circuitry. Behav. Neurol. 11, 29-42.

Bressi, C., Taylor, G., Parker, J., Bressi, S., Brambilla, V., Aguglia, E., et al. (1996). Cross validation of the factor structure of the 20-item toronto alexithymia scale: an italian multicenter study. J. Psychosom. Res.

somatoform disorder in order to defend against overwhelmingly strong emotions, can neurophysiologically be understood in terms of diminished activation of the parahippocampal gyrus. Psychotherapeutic measures aim to increase emotional insight and to accept repressed feelings and emotional memories. After psychotherapy, somatoform patients reported less symptoms and showed stronger neuronal activity in the parahippocampal gyrus. Our results support the assumption that increased access to repressed emotional memories is related to increased neuronal activity of the parahippocampal gyrus.

\section{ACKNOWLEDGMENTS}

The authors thank the staff of the Department of Neurology of the Otto-von-Guericke-University of Magdeburg for their support. We also thank Niall Duncan for his helpful propositions concerning the manuscript. Moritz de Greck had full access to all of the data in the study and takes responsibility for the integrity of the data and the accuracy of the data analysis.

\section{SUPPLEMENTARY MATERIAL}

The Supplementary Material for this article can be found online at: http://www.frontiersin.org/Human_Neuroscience/10.3389/ fnhum.2013.00410/abstract

41, 551-559. doi: 10.1016/S00223999(96)00228-0

Burba, B., Oswald, R., Grigaliunien, V., Neverauskiene, S., Jankuviene, O., and Chue, P. (2006). A controlled study of alexithymia in adolescent patients with persistent somatoform pain disorder. Can. J. Psychiat. 51, 468-471.

Cox, R. W. (1996). Afni: software for analysis and visualization of functional magnetic resonance neuroimages. Comput. Biomed. Res. 29, 162-173. doi: 10.1006/cbmr.1996.0014

Crits-Christoph, P., Cooper, A., and Luborsky, L. (1988). The accuracy of therapists' interpretations and the outcome of dynamic psychotherapy. J. Consult. Clin. Psychol. 56, 490-495. doi: 10.1037/0022006X.56.4.490

Damasio, A. R., Grabowski, T. J., Bechara, A., Damasio, H., Ponto, L. L., Parvizi, J., et al. (2000). Subcortical and cortical brain activity during the feeling of selfgenerated emotions. Nat. Neurosci. 3, 1049-1056. doi: 10.1038/79871

Davis, M. H. (1983). Measuring individual differences in empathy: evidence for a multidimensional approach. J. Pers. Soc. Psychol. 44, 113-126. doi: 10.1037/00223514.44.1.113

de Greck, M., Scheidt, L., Bölter, A. F., Frommer, J., Ulrich, C., Stockum, E., et al. (2011). Multimodal psychodynamic psychotherapy induces normalization of reward related activity in somatoform disorder. World J. Biol. Psychiat. 12, 296-308. doi: 10.3109/15622975. 2010.539269

de Greck, M., Scheidt, L., Bölter, A. F., Frommer, J., Ulrich, C., Stockum, E., et al. (2012). Altered brain activity during emotional empathy in somatoform disorder. Hum. Brain. Mapp. 33, 2666-2685. doi: 10.1002/hbm.21392

Derogatis, L. R. (1977). SCL-90$R$, Administration, Scoring and Procedures Manual-I for the R(evised) Version. Baltimore, MD: Johns Hopkins University School of Medicine

Duddu, V., Isaac, M. K., and Chaturvedi, S. K. (2003). Alexithymia in somatoform and depressive disorders. $J$. Psychosom. Res. 54, 435-438. doi: 10.1016/S0022-3999(02)00440-3

Eriksen, H. R., and Ursin, H. (2004). Subjective health complaints, sensitization, and sustained cognitive activation (stress). J. Psychosom. Res. 56, 445-448. doi: 10.1016/S00223999(03)00629-9

Franke, G. H. (2002). Die SymptomCheckliste von Derogatis, Deutsche version, Manual. Göttingen: Beltz.

Friston, K. J., Holmes, A. P., Worsley, K. J., Poline, J. P., Frith, C. D., and Frackowiak, R. S. J. (1995). Statistical parametric maps in functional imaging: a general linear approach. Hum. Brain. Mapp. 2, 189-210. doi: 10.1002/hbm.460020402 
Gardini, S., Cornoldi, C., De Beni, R., and Venneri, A. (2006). Left mediotemporal structures mediate the retrieval of episodic autobiographical mental images. Neuroimage 30, 645-655. doi: 10.1016/j.neuroimage.2005.10.012

Gemeinsamer Bundesausschuss (2009). Richtlinie des Gemeinsamen Bundesausschusses über die Durchführung der Psychotherapie. Bundesanzeiger 58:1399.

Grabe, H. J., Frommer, J., Ankerhold, A., Ulrich, C., Groger, R., Franke, G. H., et al. (2008). Alexithymia and outcome in psychotherapy. Psychother. Psychosom. 77, 189-194. doi: 10.1159/000119739

Grabe, H. J., Spitzer, C., and Freyberger, H. J. (2004). Alexithymia and personality in relation to dimensions of psychopathology. Am. J. Psychiat. 161, 1299-1301. doi: 10.1176/appi.ajp.161.7.1299

Haase, M., Frommer, J., Franke, G. H., Hoffmann, T., Schulze-Muetzel, J., Jäger, S., et al. (2008). From symptom relief to interpersonal change: treatment outcome and effectiveness in inpatient psychotherapy. Psychotherapy Res. 18, 615-624. doi: 10.1080/10503300802 192158

Hiller, W., Cebulla, M., Korn, H. J., Leibbrand, R., Röers, B., and Nilges, P. (2010). Causal symptom attributions in somatoform disorder and chronic pain. J. Psychosom. Res. 68, 9-19. doi: 10.1016/j.jpsychores.2009.06.011

Hoffmann, S. O., Hochapfel, G., Eckhardt-Henn, A., and Heuft, G. (2004). Neurotische Störungen und Psychosomatische Medizin. Stuttgart: Schattauer.

Huber, D., Albrecht, C., Hautum, A., Henrich, G., and Klug, G. (2009). [Effectiveness of inpatient psychodynamic psychotherapy: a followup study]. Z. Psychosom. Med. Psychother 55, 189-199.

Josephs, O., Turner, R., and Friston, K. (1997). Event-related fmri. Hum. Brain. Mapp. 5, 243-248.

Kirmayer, L. J., Robbins, J. M., and Paris, J. (1994). Somatoform disorders: personality and the social matrix of somatic distress. J. Abnorm. Psychol. 103, 125-136. doi: 10.1037/0021-843X.103.1.125

Krystal, H. (1997). Desomatization and the consequences of infantile psychic trauma. Psychoanal. Inq. 17, 126-150. doi: 10.1080/07351699709 534116

Leichsenring, F. (2005). Are psychodynamic and psychoanalytic therapies effective?: a review of empirical data. Int. J. Psychoanal. 86(Pt 3),
841-868. doi: 10.1516/RFEELKPN-B7TF-KPDU

Lipowski, Z. J. (1988). Somatization: the concept and its clinical application. Am. J. Psychiat. 145, 1358-1368.

Loughead, J. W., Luborsky, L., Weingarten, C. P., Krause, E. D., German, R. E., Kirk, D., and Gur, R. C. (2010). Brain activation during autobiographical relationship episode narratives: a core conflictual relationship theme approach. Psychotherapy Res. 20, 321-336. doi: 10.1080/10503300903470735

Maguire, E. A. (2001). Neuroimaging studies of autobiographical event memory. Philos. Trans. R. Soc. Lond. B Biol. Sci. 356, 1441-1451. doi: 10.1098/rstb.2001.0944

Matsumuto, D., and Ekman, P. (1988). Japanese and Caucasian Facial Expressions of Emotion (JACFEE) and Neutral Faces (JACNeuF). San Francisco, CA: University of California.

Mattila, A. K., Kronholm, E., Jula, A., Salminen, J. K., Koivisto, A. M., Mielonen, R. L., et al. (2008). Alexithymia and somatization in general population. Psychosom. Med. 70, 716-722.

Nakao, M., and Barsky, A. J. (2007). Clinical application of somatosensory amplification in psychosomatic medicine. Biopsychosoc. Med. 1:17. doi: 10.1186/1751-0759-1-17

Nichols, T., and Hayasaka, S. (2003). Controlling the familywise error rate in functional neuroimaging: a comparative review. Stat. Methods Med. Res. 12, 419-446. doi: 10.1191/0962280203sm341ra

Nickel, R., and Egle, U. T. (2001). [coping with conflict as pathogenetic link between psychosocial adversities in childhood and psychic disorders in adulthood]. Z. Psychosom. Med. Psychother. 47, 332-347.

Nickel, R., and Egle, U. T. (2006). Psychological defense styles, childhood adversities and psychopathology in adulthood. Child Abuse Negl. 30, 157-170. doi: 10.1016/j.chiabu.2005.08.016

Niki, K., and Luo, J. (2002). An fmri study on the time-limited role of the medial temporal lobe in long-term topographical autobiographic memory. J. Cogn. Neurosci. 14, 500-507. doi: 10.1162/089892902317362010

Pedrosa Gil, F., Ridout, N., Kessler, H., Neuffer, M., Schoechlin, C., Traue, H. C., et al. (2009). Facial emotion recognition and alexithymia in adults with somatoform disorders. Depress. Anxiety 26, 26-33. doi: 10.1002/da.20456
Pedrosa Gil, F., Scheidt, C. E., Hoeger, D., and Nickel, M. (2008). Relationship between attachment style, parental bonding and alexithymia in adults with somatoform disorders. Int. J. Psychiat. Med. 38, 437-451. doi: 10.2190/PM.38.4.d

Preston, S. D., and de Waal, F. B. (2002). Empathy: its ultimate and proximate bases. Behav. Brain Sci. 25, $1-20$.

Rekkas, P. V., and Constable, R. T. (2005). Evidence that autobiographic memory retrieval does not become independent of the hippocampus: an fmri study contrasting very recent with remote events. J. Cogn. Neurosci. 17, 1950-1961. doi: 10.1162/089892905775008652

Schmeing, J. B., Kehyayan, A., Kessler, H., Do Lam, A. T., Fell, J., Schmidt, A. C., et al. (2013). Can the neural basis of repression be studied in the mri scanner? new insights from two free association paradigms. PLoS ONE 8:e62358. doi: 10.1371/journal.pone. 0062358

Schur, M. (1955). Comments on the metapsychology of somatiziation. Psychoanal. Study Child 10, 119-164.

Shamay-Tsoory, S. G. (2011). The neural bases for empathy. Neuroscientist 17, 18-24. doi: $10.1177 / 1073858410379268$

Sifneos, P. E. (1973). The prevalence of 'alexithymic' characteristics in psychosomatic patients. Psychother. Psychosom. 22, 255-262. doi: 10.1159/000286529

Smith, A. P., Henson, R. N., Dolan, R. J., and Rugg, M. D. (2004). fmri correlates of the episodic retrieval of emotional contexts. 22, 868-878.

Spitzer, C., Barnow, S., Gau, K., Freyberger, H. J., and Grabe, H. J. (2008). Childhood maltreatment in patients with somatization disorder. Aust. N.Z. J. Psychiatry 42, 335-341. doi: 10.1080/00048670 701881538

Stein, D. J., and Muller, J. (2008). Cognitive-affective neuroscience of somatization disorder and functional somatic syndromes: reconceptualizing the triad of depressionanxiety-somatic symptoms. CNS Spectr. 13, 379-384.

Sterpenich, V., D'Argembeau, A., Desseilles, M., Balteau, E., Albouy, G., Vandewalle, G., et al. (2006) The locus ceruleus is involved in the successful retrieval of emotional memories in humans. J. Neurosci. 26, 7416-7423.

Talairach, J., and Tournoux, P. (1988). Co-planar Stereotaxic Atlas of the Human Brain. New York, NY: Thieme.
Vaillant, G. E. (1977). Adaptation to Life. Boston, MA: Harvard University Press.

Waller, E., and Scheidt, C. E. (2006). Somatoform disorders as disorders of affect regulation: a development perspective. Int. Rev. Psychiatr. 18, 13-24. doi: 10.1080/09540260500466774

Wittchen, H., Zaudig, M., and Fydrich, T. (1997). SKID. Strukturiertes Klinisches Interview für DSM-IV. Göttingen: Hogrefe, Verlag für Psychologie.

Witthöft, M., and Hiller, W. (2010). Psychological approaches to origins and treatments of somatoform disorders. Annu. Rev. Clin. Psychol. 6, 257-283. doi: 10.1146/annurev. clinpsy.121208.131505

Wöller, W., and Kruse, J., (eds.). (2010). Tiefenpsychologisch fundierte Psychotherapie. Stuttgart: Schattauer.

Wood, R. L., Williams, C., and Kalyani, T. (2009). The impact of alexithymia on somatization after traumatic brain injury. Brain Injury 23, 649-654. doi: $10.1080 / 02699050902970786$

Conflict of Interest Statement: The authors declare that the research was conducted in the absence of any commercial or financial relationships that could be construed as a potential conflict of interest.

Received: 21 March 2013; paper pending published: 23 May 2013; accepted: 11 July 2013; published online: 16 August 2013.

Citation: de Greck $M$, Bölter AF, Lehmann L, Ulrich C, Stockum E, Enzi B, Hoffmann T, Tempelmann C, Beutel M, Frommer $J$ and Northoff $G$ (2013) Changes in brain activity of somatoform disorder patients during emotional empathy after multimodal psychodynamic psychotherapy. Front. Hum. Neurosci. 7:410. doi: 10.3389/ fnhum.2013.00410

This article was submitted to the journal Frontiers in Human Neuroscience.

Copyright (C) 2013 de Greck, Bölter, Lehmann, Ulrich, Stockum, Enzi, Hoffmann, Tempelmann, Beutel, Frommer and Northoff. This is an open-access article distributed under the terms of the Creative Commons Attribution License (CC BY). The use, distribution or reproduction in other forums is permitted, provided the original author(s) or licensor are credited and that the original publication in this journal is cited, in accordance with accepted academic practice. No use, distribution or reproduction is permitted which does not comply with these terms. 